\title{
Langues secondes à l'école primaire au Québec
}

Reflet d'une situation de contact des langues

\section{Diane Huot}

\section{OpenEdition}

\section{Journals}

Édition électronique

URL : http://journals.openedition.org/ries/3398

DOI : 10.4000/ries.3398

ISSN : 2261-4265

Éditeur

Centre international d'études pédagogiques

Édition imprimée

Date de publication : 1 mars 1996

Pagination : 99-111

ISSN : 1254-4590

Référence électronique

Diane Huot, "Langues secondes à l'école primaire au Québec », Revue internationale d'éducation de Sèvres [En ligne], 09 | 1996, mis en ligne le 20 août 2013, consulté le 23 mars 2021. URL : http:// journals.openedition.org/ries/3398; DOI : https://doi.org/10.4000/ries.3398

Ce document a été généré automatiquement le 23 mars 2021

(C) Tous droits réservés 


\title{
Langues secondes à l'école primaire au Québec
}

\author{
Reflet d'une situation de contact des langues
}

Diane Huot

1 Le français et l'anglais sont enseignés comme langues maternelles (L1) et comme langues secondes (L2) dans les écoles du Québec. Mais compte tenu du fait que certains écoliers issus de familles d'immigrants peuvent avoir une autre langue que le français et l'anglais comme L1, cette distinction, qui ne correspond pas toujours aux caractéristiques de l'élève, évoque plutôt celles de l'école, à savoir la langue d'enseignement et la langue d'administration en usage à l'école. On parle ainsi d'«écoles françaises" et d'«écoles anglaises». Dans le premier cas, la langue d'enseignement est le français et cette langue est enseignée dans une perspective de pédagogie de L1; l'anglais y est enseigné comme L2, dans le sens où les moyens mis en œuvre pour la transmission du savoir relèvent de la pédagogie des L2. Dans le second cas, la langue d'enseignement est l'anglais et cette langue est enseignée dans une perspective de pédagogie de L1; le français y est alors enseigné comme L2.

2 Par ailleurs, cet enseignement des langues se déroule aux cours primaire et secondaire, de même qu'au collège d'enseignement général et professionnel (CEGEP). Le cours primaire a une durée de six ans (de la première à la sixième année) et il est réparti en deux cycles, soit le premier (de la première à la troisième année) et le second cycle (de la quatrième à la sixième année). La durée du cours secondaire est de cinq ans (de secondaire I à secondaire V).

3 Nous nous proposons de donner ici un aperçu de l'enseignement des L2 au primaire, à savoir l'anglais et le français. Il s'agit d'une présentation établie essentiellement à partir de documents publiés qui étaient accessibles au moment de la préparation de ce texte. Ces documents correspondent principalement aux programmes d'études actuels (en cours de révision) et aux pratiques recommandées. Il n'est pas fait mention des écarts susceptibles de se présenter dans les faits entre les recommandations des programmes et leur mise en œuvre effective par une commission scolaire, une école ou un enseignant. Mais afin de mieux comprendre les enjeux de l'enseignement des 
langues secondes et les raisons qui prévalent à son organisation, il convient d'abord de rappeler certains éléments de la situation démographique et linguistique du Québec et quelques faits saillants de la politique linguistique. Pour la présentation de telles données nous nous inspirons de G. Dumas ${ }^{1}$.

\section{Situation démolinguistique : quelques éléments}

4 La population du Québec constitue aujourd'hui $25,1 \%$ de la population canadienne (quelques vingt-huit millions), qui est très majoritairement de langue anglaise. Sur le continent nord-américain, les francophones représentent à peine $2 \%$ de la population. Selon les données du dernier recensement de 1991, le Québec compte près de sept millions de citoyens. De ce nombre $82 \%$ se sont déclarés de langue maternelle unique française, $9 \%$ de langue maternelle anglaise, $0,4 \%$ de langue autochtone et $8 \%$ de langue autre que française, anglaise et autochtone. Ce dernier groupe est dénommé "allophones». Signalons aussi que les dix nations amérindiennes et la nation Inuit (dénommées également "populations autochtones ») représentent environ $1 \%$ de la population du Québec.

5 D'autre part, le taux de fécondité des Québécois est relativement bas, car il est inférieur au nombre moyen de 2,1 enfants par femme nécessaire, selon les démographes, au renouvellement de la population. Cet indicateur met en évidence l'importance que revêt l'immigration au Québec, bien que celle-ci ait été réduite depuis quelques temps.

\section{Politique linguistique}

6 L'un des objectifs de la politique linguistique québécoise est de faire en sorte que le français devienne la langue commune de la vie publique de tous les Québécois et de favoriser le choix de cette langue d'usage par les immigrants. L'origine de ces derniers est cependant très variée. Il n'est pas rare, en effet, de voir des immigrants qui, à leur arrivée, ne savent ni le français ni l'anglais. Comme le signale J.C. Corbeil ${ }^{2}$, «ils se trouvent très souvent devant la nécessité de devoir apprendre deux langues étrangères, d'où la tentation de choisir d'abord la langue dominante sur le continent nordaméricain ». C'est pourquoi il est important d'avoir recours à un "dispositif d'intégration linguistique et culturelle des immigrants ».

\section{Charte de la langue française}

7 En 1977, le gouvernement du Québec a adopté la Charte de la langue française qui constitue le principal texte législatif régissant l'usage des langues en présence sur le territoire. Communément appelée Loi 101, la Charte de la langue française a succédé à la Loi sur la langue officielle de 1974. Elle est à la fois réalité et symbole dans le paysage linguistique québécois, mais assortie d'une dizaine de règlements d'application, elle orchestre les rapports entre le français et les différentes langues en usage au Québec. Le préambule de la Charte fait état de la volonté de « faire du français la langue de l'État et de la Loi aussi bien que la langue normale et habituelle du travail, de l'enseignement, des communications, du commerce et des affaires ». Il y est également précisé que

«L'Assemblée nationale entend poursuivre cet objectif dans un esprit de justice et

d'ouverture, dans le respect de la communauté québécoise d'expression anglaise et 
celui des minorités ethniques, dont elle reconnaît l'apport précieux au développement du Québec. L'Assemblée nationale reconnaît aux Amérindiens et aux Inuit du Québec, descendants des premiers habitants du pays, le droit qu'ils ont de maintenir et de développer leur langue et culture d'origine. »

8 La politique linguistique qui consacre le français comme la langue officielle du Québec réserve ainsi une place importante à deux objectifs fondamentaux: accorder au français la place qui lui revient dans la vie économique et diriger les immigrants vers l'école française. La réalisation de ces objectifs devait permettre au français de devenir et de demeurer la langue commune au Québec.

Une telle démarche s'inscrit dans la situation numériquement précaire des francophones dans l'ensemble nord-américain, où il devenait impératif que le gouvernement du Québec légifère de manière à assurer l'utilisation de la langue française en de multiples fonctions. En conséquence, la Charte de la langue française compte parmi les quelques législations linguistiques qui touchent également un éventail aussi large de domaines. Ces dispositions régissent, à divers degrés, les droits linguistiques fondamentaux, la langue de la législation et de la justice, de l'administration publique et des organismes parapublics, de l'enseignement, du travail, du commerce et des affaires. De plus, le législateur a institué trois organismes dont les mandats concourent à l'application de la politique linguistique. Il s'agit du Conseil de la langue française, de la Commission de toponymie et de l'office de la langue française. Ainsi, parallèlement à des domaines comme ceux du travail, du commerce ou des affaires, la langue d'enseignement est également régie par cette législation linguistique.

\section{Langue d'enseignement et fréquentation des « écoles françaises »}

10 À l'exception des niveaux d'études collégial et universitaire, qui ne sont pas visés par la Charte, les élèves et étudiants de ces niveaux pouvant faire leurs études supérieures dans la langue de leur choix, le français est de manière générale la langue de l'enseignement au Québec. Cette exigence ne vaut cependant pas pour les populations autochtones qui ne sont pas régies par la Charte. Quant au droit à l'éducation en anglais au Québec, il est reconnu uniquement à certaines catégories d'élèves qui satisfont à des exigences précises stipulées dans la Charte, ces catégories d'élèves bénéficiant de droits historiques de recevoir l'enseignement dans leur langue.

\section{Accueil des immigrants}

En établissant de tels critères d'admission, le législateur visait à amener les enfants des immigrants et des francophones à l'« école française ». À cet égard, on peut considérer aujourd'hui que la Charte a produit des résultats probants. Lors de son adoption en 1977, seulement $20 \%$ des élèves allophones fréquentaient l'école française, tandis qu'en 1995, on en comptait $80 \%$.

\section{Programme d'enseignement des langues d'origine}

Un autre élément qu'il faut également signaler est l'existence du Programme d'enseignement des langues d'origine (PELO), mis sur pied en 1977, soit l'année de l'adoption de la Charte de la langue française. Ce programme s'inscrit dans le prolongement de la Charte des droits et libertés de la personne selon laquelle «les 
personnes appartenant à des minorités ethniques ont le droit de maintenir et de faire progresser leur propre vie culturelle avec les autres membres de leur groupe ». Il est offert par l'école publique en une douzaine de langues, pendant ou après les heures de classe, à raison de trente minutes par jour. De nombreuses recherches soutiennent que la consolidation de la langue d'origine est un des facteurs facilitant de manière sensible l'apprentissage de toutes les matières enseignées, incluant la langue seconde. De plus, il semble que lorsque l'école valorise et accepte la culture des jeunes immigrants, l'élève est mieux disposé à apprendre la langue du milieu d'accueil.

\section{Enseignement des langues secondes}

13 On peut voir à travers la présentation qui précède de ces quelques éléments de la situation démolinguistique et de la politique linguistique que l'école primaire québécoise poursuit des objectifs importants en matière de pédagogie des langues secondes, que ce soit à l'« école anglaise » fréquentée par les anglophones, ou à l'« école française ", fréquentée par les francophones et les immigrants. Tout en respectant les exigences de la législation, l'école primaire doit ainsi assurer, d'une part, l'enseignement $d u$ français langue seconde aux anglophones et aux immigrants non anglophones et, d'autre part, l'enseignement de l'anglais langue seconde à tous les enfants non anglophones. Ces précisions étant faites, voyons comment de tels objectifs se traduisent dans l'organisation de l'enseignement du français et de l'anglais langues secondes à l'école primaire.

\section{Enseignement du français $L 2$ au primaire}

14 Pour répondre aux besoins des enfants qui constituent, selon le dernier recensement de 1991 , ce $9 \%$ de la population de langue maternelle anglaise et ce $8 \%$ de langue autre que française, anglaise et autochtone, il était nécessaire, d'un point de vue pédagogique, d'adopter des mesures différentes, appropriées à ces deux catégories d'enfants. L'enseignement du français L2 est ainsi réparti en deux secteurs distincts, à savoir celui des classes de français L2 des "écoles anglaises» et celui des classes d'accueil et de francisation des « écoles françaises ».

La différence essentielle entre ces deux secteurs réside dans la nature des publics visés et l'objectif poursuivi. Dans le premier cas, l'élève inscrit à l'« école anglaise » va de manière générale continuer à recevoir, à l'exception du français L2, un enseignement en anglais pendant toute la durée de son cours primaire et secondaire. Dans le second cas, l'élève inscrit à l'« école française " sera par la suite intégré à l'enseignement régulier en français. Il reçoit ainsi pendant un certain temps un enseignement du français effectué selon une perspective de pédagogie des L2, après quoi il sera accueilli dans le système régulier où il recevra un enseignement en français de toutes les matières, dont le cours de français effectué selon une perspective de pédagogie de L1.

\section{Classes de français $L 2$ des « écoles anglaises »}

Le Régime pédagogique ${ }^{3}$ prévoit que dans le cas des écoles primaires anglophones, l'étude du français L2 commence à partir de la première année du primaire, à raison de deux heures par semaine. Mais il semble, d'après les résultats d'un sondage, qu'un bon 
nombre d'élèves soient exposés à plus de cent vingt minutes par semaine de français L2.

D'autre part, on trouve plusieurs modèles d'organisation de l'enseignement du français L2 ${ }^{4}$ qui peuvent être regroupés en deux catégories, à savoir des modèles offrant uniquement le français langue seconde en français et des modèles offrant d'autres matières en français. Ces renseignements sont issus d'une étude ${ }^{5}$ dont le taux de répondants constituait, au moment de la collecte des données, $9 \%$ de la population scolaire totale.

\section{Catégorie des modèles offrant uniquement le français, langue seconde, en français}

Cette catégorie comprend le Core French et le Enriched Core. Le modèle Core French correspond à une organisation du temps d'enseignement du français L2 qui varie entre quatre-vingt-dix et cent cinquante minutes par semaine. L'élève ayant suivi des cours dans le cadre de ce modèle aura reçu entre trois cents et cinq cents heures de français L2 pendant les six années du primaire. Le modèle Enriched Core offre de cent cinquantecinq à trois cents minutes de français L2 par semaine, ce qui équivaut pour l'ensemble du primaire à environ mille heures de français L2.

\section{Catégorie des modèles offrant d'autres matières en français}

Cette catégorie regroupe l'Extended Core, l'Intensification de 28 à $50 \%$ de la scolarité et l'Intensification de 51 à $82 \%$ de la scolarité. Selon le modèle appelé Extended Core, l'élève reçoit de cent quarante à trois cent soixante minutes de français par semaine, soit des cours de français L2 et un enseignement en français d'une ou de deux autres matières, et ce pendant un ou deux cycles. Ce modèle permet à l'élève de faire de cinq cents à mille deux cents heures en français pendant toute la durée du cours primaire. L'Intensification de 28 à $50 \%$ de la scolarité signifie que le temps d'enseignement du français est de quatre cents à sept cent cinq minutes par semaine, soit un total de mille quatre cents à deux mille cinq cents heures pendant toute la durée du primaire. Elle se déroule selon quatre types d'organisation appelés «intensification constante ou presque constante pendant un ou deux cycles », «immersion partielle au début du premier cycle », « immersion partielle ou totale au deuxième cycle » et " année 6+ » qui comporte une année supplémentaire d'activités en français.

20 L'Intensification de 51 à $82 \%$ de la scolarité comporte des formules où une moyenne de sept cent dix à mille cent soixante minutes de français sont offertes chaque semaine, ce qui correspond à un total d'environ deux mille cinq cents à quatre mille heures pendant le cours primaire. Toujours selon Campbell ${ }^{6}$, ce type d'intensification peut se dérouler selon quatre types d'organisation différents soit l'intensification constante ou presque constante pendant deux cycles; l'immersion partielle au premier cycle avec diminution après; l'immersion partielle au premier cycle avec augmentation au deuxième cycle et l'immersion totale au premier cycle pendant une période de un à trois ans avec diminution après.

21 Comme on peut le voir, ces catégories de modèles offrent des conditions d'appropriation fort différentes, surtout en ce qui a trait au temps alloué à l'apprentissage. La question de la relation entre ces diverses conditions d'appropriation et les résultats obtenus par les élèves dans chacun des modèles évoqués dépasse cependant les limites de ce texte. Des études sont en cours et, à notre connaissance, 
l'une d'entre elles ${ }^{7}$ qui porte sur l'analyse des performances en français écrit d'élèves non francophones du primaire paraitra incessamment.

Modèles d'enseignement du français L2 dans les « écoles anglaises » (selon Campbell)

\begin{tabular}{|c|c|c|c|}
\hline Modèles & $\begin{array}{l}\text { Nombre de minutes } \\
\text { d'enseignement } \\
\text { par semaine }\end{array}$ & $\begin{array}{c}\text { Nombre total d'heures } \\
\text { de français pendant } \\
\text { toute la durée } \\
\text { du cours primaire }\end{array}$ & $\begin{array}{c}\text { Pourcentage } \\
\text { de la population scolaire } \\
\text { par catégorie (taux } \\
\text { de réponses } 90 \% \text { ) }\end{array}$ \\
\hline \multicolumn{4}{|l|}{$\begin{array}{l}\text { 1. Modèles offrant } \\
\text { uniquement le français, } \\
\text { langue seconde } \\
\text { en français }\end{array}$} \\
\hline Core French & 90 à 150 & 300 à 500 & $16 \%$ \\
\hline Enriched Core & 155 à 300 & 500 à 1000 & $20 \%$ \\
\hline \multicolumn{4}{|l|}{$\begin{array}{l}\text { 2. Modèles offrant } \\
\text { d'autres matières } \\
\text { en français }\end{array}$} \\
\hline Extended Core & $\begin{array}{l}140 \text { à } 360 \text { de français L2 } \\
\text { + étude en français } \\
\text { de } 1 \text { ou } 2 \text { matières } \\
\text { pendant } 1 \text { ou } 2 \text { cycles }\end{array}$ & 500 à 1000 pendant 6 ans & $15 \%$ \\
\hline $\begin{array}{l}\text { Intensification de } 28 \text { à } \\
50 \% \text { de la scolarité } \\
\text { (4 types d'organisation) }\end{array}$ & 400 à 705 & 1400 à 2500 & $24 \%$ \\
\hline $\begin{array}{l}\text { Intensification de } 51 \text { à } \\
82 \% \text { de la scolarité } \\
\text { (4 types d'organisation) }\end{array}$ & 710 à 1160 & 2500 à 4000 & $25 \%$ \\
\hline
\end{tabular}

\section{Programme d'études et approches d'enseignement}

L'enseignement du français L2 dans les écoles anglophones doit suivre les directives énoncées dans le Programme d'études de français, langue seconde ${ }^{8}$. Ce programme est de type communicatif. Il a été implanté dans les écoles à partir de 1986 de manière à rendre les élèves aptes, à la fin du secondaire, à pouvoir communiquer en français dans les situations où l'usage du français s'impose. Ce choix d'ordre pédagogique signifie que l'enseignement du français va favoriser une appropriation de la L2 dans la classe à l'aide d'interactions. Il est contextualisé, motivant et principalement axé sur le sens du message. Les manuels agréés par le ministère de l'Éducation répondent aux exigences de ce programme communicatif.

\section{Classes de français pour les non-anglophones : accueil et francisation dans les " écoles françaises "}

Les immigrants non anglophones sont dirigés vers les écoles françaises. Le Régime

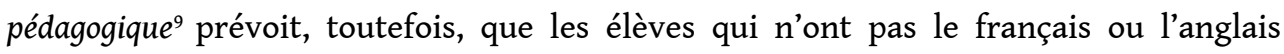
comme langue maternelle peuvent bénéficier de "services d'aide à l'intégration scolaire et sociale et à l'apprentissage de la langue française ». De tels services comprennent les services d'accueil, de francisation et de soutien linguistique.

Les services d'accueil, dispensés au moyen de classes ou d'autres mesures d'accueil, sont destinés à l'élève «qui est inscrit à l'enseignement en français pour la première 
fois, qui est inadmissible à l'enseignement en anglais selon la loi, qui réside au Québec depuis moins de cinq ans [...] et dont la connaissance de la langue française ne lui permet pas de suivre normalement les cours dans une classe ordinaire ". Les élèves bénéficiant de ce type de services reçoivent la plupart du temps un enseignement de français L2 en français, adapté à leurs besoins respectifs. De telles mesures leur fournissent la préparation nécessaire à leur intégration ultérieure au système d'enseignement régulier.

Les services de francisation, dispensés au moyen de classes ou d'autres mesures spéciales de francisation, s'adressent à l'élève « inadmissible aux services d'accueil, qui est inscrit à l'enseignement en français pour la première fois et dont la connaissance de la langue française ne lui permet pas de suivre normalement les cours dans une classe ordinaire ». Comme dans le cas précédent, il s'agit d'une aide adaptée aux besoins spécifiques de l'élève qui doit se préparer à passer au système régulier.

Les services de soutien linguistique sont prévus pour les élèves «inscrits à l'enseignement en français dans une classe ordinaire et ayant bénéficié de services d'accueil ou de francisation ». De telles mesures sont à la disposition des élèves inscrits dans le système régulier et qui éprouveraient encore quelques difficultés à fonctionner avec les francophones.

\section{Programme d'étude et approches d'enseignement pour les services d'accueil et de francisation}

Un programme d'études, Programme d'études. Français, classe d'accueil classe de francisation $^{10}$, a été élaboré pour répondre aux besoins des élèves d'accueil et de francisation. Par ses objectifs langagiers et socio-culturels, il « vise à donner à l'élève non-francophone les moyens de pouvoir "communiquer son expérience» et de s'ouvrir sur «le monde, sur les personnes et sur les choses, en vue d'en découvrir la nature et la diversité ». Il s'agit d'un programme de type communicatif dans lequel la L2 est vue comme un moyen de communication et un véhicule des valeurs socioculturelles. Il préconise une "pédagogie du savoir-faire et de l'intégration des habiletés, qui tient compte du développement de l'élève et qui respecte la culture d'origine et la manière de vivre de celui-ci ».

\section{Enseignement de l'anglais $\mathrm{L} 2$ au primaire}

L'étude de l'anglais L2 est nécessaire pour tous les élèves non francophones. Le Régime pédagogique ${ }^{11}$ prévoit, à cet effet, que dans le cas des écoles primaires francophones, l'étude de l'anglais L2 est obligatoire à partir de la $4^{\mathrm{e}}$ année, à l'exception des " projets d'innovation pédagogiques autorisés par le ministre ». Un commentaire à ce régime pédagogique stipule par ailleurs qu'une commission scolaire donnée peut répartir à son choix ces cours d'anglais au deuxième cycle du primaire, dans la mesure où les moyens sont pris pour « assurer la réalisation des objectifs obligatoires du programme ».

\section{Programme d'études et approches d'enseignement}

Selon le Programme d'études ${ }^{12}$ actuellement en vigueur, l'objectif global de l'enseignement de l'anglais L2 est d'amener l'élève non anglophone à pouvoir « utiliser la langue anglaise comme moyen de communication dans des situations correspondant 
à des besoins et à des intérêts propres à son âge ». Deux objectifs généraux découlent de cet objectif global, à savoir un premier qui a trait à la compréhension orale et écrite : «l'élève sera capable de comprendre les messages qui lui sont adressés, oralement ou par écrit, dans un nombre limité de situations que vivent les enfants anglophones du même âge ", et un second qui concerne l'expression orale et écrite: "l'élève sera capable de s'exprimer et de se faire comprendre, oralement ou par écrit, dans un nombre limité de situations que vivent les enfants anglophones du même âge ».

Ce programme implique un enseignement de type communicatif, car «il est conçu à partir de l'utilisation fonctionnelle de la langue plutôt qu'à partir de ses formes grammaticales». Comme dans le cas du français L2, un tel choix signifie que l'enseignement de l'anglais L2 va favoriser une appropriation dans la classe à l'aide d'interactions. Il est contextualisé, motivant et principalement axé sur le sens du message. Les manuels agréés par le ministère de l'Éducation doivent refléter ce choix d'ordre pédagogique. Ce programme étant en cours de révision, il est possible que certains de ces éléments changent dans un avenir prochain.

\section{Temps d'enseignement}

Dans un mémoire présenté à la Commission des États généraux sur l'éducation, la Société pour la promotion de l'enseignement de l'anglais, langue seconde, au Québec (SPEAQ) signale, que selon le programme du ministère de l'Éducation, le temps alloué à l'enseignement de l'anglais L2 à l'école primaire devrait comporter un minimum de deux cent seize heures. Le tableau suivant tiré de Speak out ${ }^{13}$ présente la répartition souhaitée de ces heures d'enseignement.

Enseignement de l'anglais L2 au primaire

\begin{tabular}{|l|l|}
\hline $4^{\mathrm{e}}$ année & $\begin{array}{l}\text { un minimum de } 72 \text { heures } \\
120 \text { minutes/semaine }\end{array}$ \\
\hline $5^{\mathrm{e}}$ année & $\begin{array}{l}\text { un minimum de } 72 \text { heures } \\
120 \text { minutes/semaine }\end{array}$ \\
\hline $6^{\mathrm{e}}$ année & $\begin{array}{l}\text { un minimum de } 72 \text { heures } \\
120 \text { minutes/semaine }\end{array}$ \\
\hline
\end{tabular}

Mais dans les faits, il est possible que cet objectif d'un total de deux cent seize heures ne soit pas atteint de la même façon dans toutes les commissions scolaires, diverses raisons pouvant contribuer parfois à réduire le nombre d'heures consacré à l'enseignement de l'anglais L2. Les représentants de la SPEAQ rappelaient du reste au ministre de l'Éducation que

«l'octroi d'un minimum de deux cent seize heures pour l'enseignement de l'anglais, langue seconde, au primaire constitu [ait] une priorité $»^{14}$. Ils ajoutaient également que « l'obligation de dispenser deux cent seize heures au second cycle du primaire aidera à éliminer les grandes disparités qui existent actuellement d'une commission scolaire à une autre, voire d'une école à une autre. Ces disparités font que beaucoup d'élèves arrivent au secondaire sans posséder les bases nécessaires pour le programme du premier cycle du secondaire. Ils accusent déjà un retard important qu'ils ne rattrapent que difficilement, et parfois jamais. » 


\section{Modalités d'enseignement}

Deux formules principales ont cours pour l'enseignement de l'anglais L2, à savoir l'enseignement dit «régulier » et l'enseignement dit «intensif», lequel comporte plusieurs versions selon les pratiques.

L'enseignement "régulier" correspond aux exigences du programme quant à la répartition du temps d'enseignement, à savoir cent vingt minutes par semaine. Le contenu du programme d'études est celui qui est recommandé dans le programme de base. Mais dans les faits, cette formule comporte, selon les écoles et les commissions scolaires, plusieurs variantes quant à la répartition du temps. On trouve, par exemple, des cas où l'ensemble des heures d'enseignement est regroupé dans certaines parties de l'année, ou encore d'autres cas où on offre un nombre plus élevé d'heures d'anglais L2 que celui prévu dans le régime pédagogique.

4 Par ailleurs, l'enseignement « intensif » se définit par la quantité de temps consacré à l'anglais L2 et par la concentration de ce temps. W. Watts et S. Snow ${ }^{15}$ parlent d'intensif dans le cas de «tous les projets qui accordent plus de $40 \%$ du temps d'enseignement total à l'enseignement de l'anglais langue seconde ».

Ces auteurs s'appuient sur une étude menée en $1987-1988^{16}$ dont l'objectif était de recenser dans la province de Québec l'ensemble des programmes qui allouaient à l'enseignement de l'anglais L2 un nombre plus élevé d'heures que celui qui était recommandé par le ministère de l'Éducation. Parmi les types de programmes alors recensés, l'enseignement intensif présente un intérêt particulier. Cependant, W. Watts et $\mathrm{S}$. Snow ${ }^{17}$ constatent que

«le nombre de groupes d'anglais intensif par rapport au nombre total de groupes

d'un même degré d'enseignement varie grandement d'une commission scolaire à

une autre ».

À titre d'exemple, ils signalent que dans une commission scolaire (Commission scolaire de l'Eau-Vive) « $2 \%$ de la clientèle de quatrième année est inscrite dans un projet d'anglais intensif, » tandis que dans d'autres cas, ce pourcentage est plus élevé, comme à la Commission scolaire de Morilac, où « $66 \%$ des groupes de $5^{\mathrm{e}}$ année reçoivent l'enseignement de l'anglais intensif » ou encore à la Commission scolaire de Greenfield Park, où ce pourcentage correspond à « $75 \%$ des groupes de $5^{\mathrm{e}}$ année ».

7 L'intensif comporte actuellement plusieurs formules d'organisation du temps dont celle de «cinq mois/cinq mois» ou de "quatre mois/six mois». On trouve aussi d'autres variantes à ces formules comme celle d'une demi-journée d'anglais à raison de cinq jours par semaine, celle d'une demi-journée d'anglais à raison de quatre jours par semaine, celle d'une semaine d'anglais et d'une semaine d'enseignement de disciplines autres que l'anglais ${ }^{18}$.

8 La formule la plus courante est celle de " cinq mois/cinq mois ». Elle correspond à une alternance dans la langue d'enseignement, une partie de l'année scolaire se déroulant dans une langue, l'autre partie se déroulant dans l'autre langue. Ainsi, l'enseignement peut se dérouler d'abord en anglais pendant une période de cinq mois, à l'exception de trois heures par semaine qui ont lieu en français pour l'enseignement des spécialités (éducation physique et musique). Dans l'autre moitié de l'année scolaire, l'enseignement se déroule ensuite entièrement en français, le programme d'anglais ayant été couvert pour l'année scolaire. 
39 La formule de « quatre mois/six mois » correspond également à une alternance dans la langue d'enseignement. Mais dans ce cas, l'élève est exposé à quatre mois d'enseignement en anglais à l'exception des trois heures pour les spécialités qui sont enseignées en français. Il reçoit ensuite son enseignement en français pendant les six autres mois de l'année.

Notons également que dans le cas de l'ensemble de ces formules, le temps de classe consacré à l'étude des matières autres que l'anglais L2 est réduit. L'élève doit ainsi remédier à cette situation en consacrant plus de temps à ses études à la maison.

41 L'intensif est accueilli favorablement par les représentants de la SPEAQ qui suggèrent du reste de "mettre en veilleuse l'idée d'organiser des classes dites d'immersion ». Une consultation menée auprès de divers groupes semble, en effet, indiquer qu'il est préférable de continuer sur la voie de l'intensif dont les résultats apparaissent intéressants. Et toujours selon ces auteurs, il semble que les "provinces canadiennes qui font l'immersion en français commencent à s'intéresser beaucoup à nos programmes intensifs $»^{19}$.

\section{Des contextes nombreux et variés}

42 L'enseignement de l'anglais L2 se déroule ainsi selon plusieurs formules et de manière différente selon les commissions scolaires et les écoles. Il se réalise aussi dans un territoire très étendu et dans des contextes aussi nombreux que variés. Il a lieu dans des régions et villes unilingues francophones, comme Charlevoix, le Bas-du-Fleuve, Rivière-Du-Loup, Québec ou Chicoutimi, où l'anglais oral est accessible par l'intermédiaire de la radio et de la télévision. Il a lieu aussi dans l'Ile de Montréal où, selon les parties de la ville, le milieu peut offrir à l'élève des occasions d'être exposé à la L2. Les résultats obtenus quant à l'apprentissage de l'anglais varient ainsi selon les circonstances et selon les apprenants.

43 Mais si les conditions créées par le milieu sont différentes selon les régions du Québec, le programme d'études est le même pour tous, les directives sont uniformes et la politique linguistique s'applique à tous les citoyens, quelle que soit la région où ils vivent. C'est pourquoi certains parents francophones expriment parfois leur insatisfaction quant au nombre d'heures réservé à l'apprentissage de l'anglais L2. Certains d'entre eux essaient même de remédier à cette situation en augmentant le nombre d'heures d'exposition à la L2 pour leurs enfants, à l'aide de camps d'été de langue anglaise. Mais il serait préférable que l'école arrive à accomplir une telle tâche. Certains souhaitent, en somme, un juste équilibre entre des objectifs de protection du français et des exigences relatives aux conditions d'appropriation d'une L2.

Il va sans dire que le déroulement d'un enseignement de langues dans un contexte multilingue comporte de nombreuses difficultés d'organisation et de pédagogie. Mais l'ensemble de ce processus est dominé par un souci constant du respect des droits, une forte volonté de contribuer à l'avenir du français en Amérique et un objectif de préparer le citoyen de demain à évoluer dans un monde où l'anglais occupera une place de grande importance. Chaque situation commande sa propre solution. L'avenir seul jugera de la pertinence de tous les choix que nous venons d'évoquer dans le cadre de ce texte. 


\section{NOTES}

1. La politique linguistique du Québec: plus de deux siècles de persévérance. Présentation faite au colloque «Culture, langue et patrimoine », UNESCO/La Sorbonne, 6-7 mars 1995, gouvernement du Québec, Secrétariat à la politique linguistique (document non publié).

2. Bilan de la situation linguistique au Québec. Légitimité et conditions de succès de l'aménagement linguistique $d u$ Québec. Contribution à l'intention du Secrétariat à la politique linguistique (document non publié), Québec, gouvernement du Québec, 1996.

3. Ministère de l'Éducation du Québec ( $\mathrm{MEQ})$, Régime pédagogique éducation préscolaire et enseignement primaire, gouvernement du Québec, 1981.

4. Voir tableau.

5. M. Campbell, Français langue seconde. Situation de l'enseignement du français langue seconde, dans les écoles anglophones du Québec, Gouvernement du Québec, ministère de l'Éducation, direction des services éducatifs aux anglophones, 1990.

6. Voir note 5, op. cit.

7. F. Lemonnier, Z. De Koninck, Étude de la performance en français écrit d'élèves non francophones de $6^{e}$ du primaire au Québec, Rapport de recherche, Québec, ministère de l'Éducation (à paraître).

8. Ministère de l'Éducation du Québec (MEQ), direction des programmes, service du primaire, 1980.

9. Voir note 3, op. cit.

10. Ministère de l'Éducation du Québec, direction des programmes, service du primaire, 1984.

11. Voir note 3, op. cit.

12. Ministère de l'Éducation du Québec (MEQ), direction des programmes, service du primaire, Programme d'études. Primaire. Anglais langue seconde, 1981.

13. M. Bonneville, Speak out, Revue pédagogique de la Société pour la promotion de l'enseignement de l'anglais, langue seconde, au Québec, 1995, 24, 1, p 4.

14. A. Pridmore, Speak out, Revue pédagogique de la Société pour la promotion de l'enseignement de l'anglais, langue seconde, au Québec, 1994, 22, 1, p. 4.

15. «L'anglais intensif au Québec », 1976-1993, Québec, Speak out, 1993, 1.

16. P. Lightbown, H. Conan, L. Bosduc, S. Guay, L'enseignement accru de l'anglais, langue seconde, dans les écoles primaires de langue française du Québec, Montréal, SPEAQ, 1988.

17. Voir note 15, op. cit.

18. Voir note 15 , op. cit.

19. Voir note 14 , op. cit.

\section{RÉSUMÉS}

Le français et l'anglais sont enseignés comme langue 2 avec des régimes pédagogiques qui prévoient des programmes d'études et des temps d'enseignement très précis pour favoriser l'intégration, notamment des immigrants, et répondre au mieux à la situation linguistique du pays. 
INDEX

Index géographique : Québec, Canada

Mots-clés : école primaire, enseignement des langues, langue anglaise, langue française, langues étrangères, langue seconde, intégration scolaire, immigration

\section{AUTEUR}

\section{DIANE HUOT}

Professeur, université Laval, Québec, Canada. 